\title{
Properties of ultraviolet lines observed with the Coronal Diagnostic Spectrometer (CDS/SOHO) in coronal holes and the quiet Sun
}

\author{
K. Stucki ${ }^{1,5}$, S. K. Solanki ${ }^{2}$, C. D. Pike ${ }^{3}$, U. Schühle ${ }^{2}$, I. Rüedi ${ }^{4}$, A. Pauluhn ${ }^{1,6,7}$, and A. Brković ${ }^{1}$ \\ 1 Institute of Astronomy, ETH-Zentrum, 8092 Zürich, Switzerland \\ 2 Max-Planck-Institut für Aeronomie, 37191 Katlenburg-Lindau, Germany \\ 3 Rutherford Appleton Laboratory, Chilton, Didcot, Oxon, OX11 0QX, UK \\ 4 PMOD/WRC, CH-7260 Davos Dorf, Switzerland \\ 5 Swiss Air Force, Ueg/RLT, 6055 Alpnach, Switzerland \\ 6 INTEC HTA Bern, Switzerland \\ 7 International Space Science Institute, Bern, Switzerland
}

Received 28 May 2001 / Accepted 10 September 2001

\begin{abstract}
We present an analysis of 14 ultraviolet emission lines belonging to different atoms and ions observed inside polar coronal holes and in the normal quiet Sun. The observations were made with the Coronal Diagnostic Spectrometer (CDS) onboard the Solar and Heliospheric Observatory (SOHO). This study extends previous investigations made with the Solar Ultraviolet Measurements of Emitted Radiation (SUMER) spectrometer to higher temperatures. We compare line intensities, shifts and widths in coronal holes with the corresponding values obtained in the quiet Sun. While all lines formed at temperatures above $7 \times 10^{5} \mathrm{~K}$ show clearly the presence of the hole in their intensities, differences in line width are more subtle, with cooler lines being broader in coronal holes, while hotter lines tend to be narrower. According to the present data all lines are blueshifted inside the coronal hole compared to the normal quiet Sun. Almost all the lines formed between $80000 \mathrm{~K}$ and $600000 \mathrm{~K}$ (i.e. transition-region lines) show a correlation between blueshifts and brightness within coronal holes. This is in agreement with the conclusion reached by Hassler et al. (1999) that the fast solar wind emanates from the network and supports our previous study (Stucki et al. 2000b). For coronal lines, this trend seems to be reversed.
\end{abstract}

Key words. Sun: corona - solar wind - transition region - UV radiation

\section{Introduction}

The plasma conditions in solar coronal holes are of considerable interest since the fast solar wind originates in these regions (Krieger et al. 1973; Leer \& Holzer 1985; Woch et al. 1997; Wilhelm et al. 2000). Coronal holes may also be of importance in the formation of the slow solar wind (Bravo \& Stewart 1997). Knowledge of the structure of coronal holes, but also of the chromosphere below them, is necessary to understand the solar wind acceleration mechanisms and coronal heating (McKenzie et al. 1998).

The corona above coronal holes is easily distinguishable from normal quiet Sun areas by its reduced brightness but also by the characteristic structure of polar plumes seen in coronal emission lines (e.g. Ahmad \& Withbroe 1997; Feldman et al. 2000). The elemental composition in the coronal layers of coronal holes as compared to that in the normal quiet Sun (which in the following we simply

Send offprint requests to: K. Stucki, e-mail: katja.stucki@lw.admin.ch refer to as quiet Sun) has been studied by Feldman et al. (1998). Tu et al. (1998) derived ion temperatures in a polar coronal hole from line width measurements. At the solar limb in polar coronal holes it was seen that the transition region is broader than in quiet Sun areas (Huber et al. 1974), and spicules and macrospicules extend farther above the photospheric limb (Wilhelm 2000b). On the solar disk, however, the differences between quiet Sun and coronal holes are not so clearly discernible in the lower corona and much less in the transition region and chromosphere. Differences in the centre-to-limb variation of the radiance of some transition region and coronal lines have been found by Wilhelm et al. (1998). Doppler shifts of C II, O VI and Ne VIII lines have been measured by Warren (1997) and of some other lines by Peter \& Judge (1999). They found that whereas cooler lines exhibit a net redshift, lines formed above $5 \times 10^{5} \mathrm{~K}$ show a net blue shift in coronal hole areas, which could be associated with a net outflow of coronal plasma. This solar wind outflow correlates with the underlying chromospheric network (Hassler et al. 1999). 
In previous studies (Stucki et al. 2000a,b) we analyzed data from the Solar Ultraviolet Measurements of Emitted Radiation (SUMER) spectrometer onboard the Solar and Heliospheric Observatory (SOHO). We found that with increasing formation temperature, spectral lines showed on average an increasingly stronger blueshift in coronal holes relative to the quiet Sun at equal heliospheric angle, with the coolest lines from our sample (formation temperature $\approx 10^{4} \mathrm{~K}$ ) indicating a small relative redshift. The width of the lines was generally larger (by a few kilometers per second) inside the coronal hole. Intensity measurements clearly showed the presence of the coronal hole in the hotter lines while intensity histograms exhibited distinct differences between coronal hole and quiet-Sun data.

Within coronal holes, lines showing the network and formed above $30000 \mathrm{~K}$ displayed a positive correlation between blueshifts and brightness (extending and supporting the conclusion reached by Hassler et al. 1999). In the normal quiet Sun, however, we found that only lines formed above $2-3 \times 10^{5} \mathrm{~K}$ showed such a trend, the cooler lines being more redshifted in the network. This suggests that either there is a fundamental difference between coronal holes and the normal quiet Sun in the initial acceleration of the solar wind, or that the wavelength-shift versus brightness relationship in the quiet Sun stems from other processes or structures (closed magnetic loops) than in coronal holes (open field lines).

The main limitations of the previously analyzed data are the limited spatial sampling (the data were obtained after the anomaly in SUMER's scan mechanism) and the lack of reliable coronal data (the only coronal line in the previous sample, FexiI $1242.01 \AA$ is both weak and strongly blended). To check the trends observed with the few available coronal lines, we needed to use an instrument providing more lines formed at higher temperatures, but still having good photon counting statistics.

Here we analyze observations of coronal holes and the quiet Sun recorded with the Normal Incidence Spectrometer (NIS) of CDS (Harrison et al. 1995) onboard SOHO. The spectral ranges of NIS (310 to $380 \AA$, and 517 to $633 \AA$ ) include lines that are formed over a large temperature range (approximately $10^{4}$ to $2 \times 10^{6} \mathrm{~K}$ ). Images of the Sun at wavelengths arising from the upper chromosphere, the transition region and the corona can be formed simultaneously by carrying out raster scans. Therefore, these data are ideally suited to complement our previous studies by providing "hotter" lines and a larger area to be sampled. CDS data suffer, however, from lower spatial and, in particular, spectral resolution.

\section{Observations, data reduction and analysis}

\subsection{Observational data}

The spectral pixel size of the CDS NIS ranges from $0.070 \AA$ at $310 \AA$ to $0.118 \AA$ at $630 \AA$. The effective pixel size of CDS for these data is $4^{\prime \prime}$ in the horizontal (cross slit) direction and $1.684^{\prime \prime}$ vertical (along slit), although the actual spatial resolution of the telescope is lower (Thompson 1998; Haugan 1999).

The analyzed datasets consist of raster scans over a certain region on the solar disk with a spectrum recorded at every spatial position of the slit. The first set, called s6159r00 was taken on December 15, 1996, as part of JOP055, using slit 5 (secondary slit, $4^{\prime \prime}$ by $240^{\prime \prime}$ ). 60 scan steps of $4^{\prime \prime}$ cover an area of $240^{\prime \prime}$ by $240^{\prime \prime}$ over the northern polar coronal hole and include 15 EUV emission lines (exposure time $60 \mathrm{~s}$ ).

The second set, s6936, was recorded on February 9, 1997. It covers an area of $240^{\prime \prime}$ by $240^{\prime \prime}$ over the southern polar coronal hole. Several identical rasters were taken using slit 4 (primary slit, $2^{\prime \prime}$ by $240^{\prime \prime}, 120$ steps of $2^{\prime \prime}$ ), including 12 different spectral lines (exposure time $40 \mathrm{~s}$ ). In this study we used the first two rasters: s6936r00 and s6936r01.

Figures 1 and 2 show the Sun as seen by EIT (Extremeultraviolet Imaging Telescope, on SOHO) in Fe XII $195 \AA$ at the time of the recording of the first and second sets of observations, respectively. The CDS rasters are outlined.

\subsection{Data reduction}

The standard CDS software was used to remove cosmic ray hits, to correct for the CCD readout bias (which may also include a scattered light component) and to do the flatfielding. In the next step, the VDS (Viewfinder Detector Subsystem, i.e. the detector used within the CDS-NIS) detector calibration is applied. It consists of the radiometric calibration and the wavelength calibration of the NIS spectrograph. Standard spectral line tilt or rotation corrections turned out to be unsatifactory for our purpose. Hence, we developed and applied our own correction for the variation of spectral line shift and width across the detector and in time, as described in the Appendix. After the instrumental corrections and radiometric calibrations, we used bilinear interpolation to fill-in missing pixels due to telemetry dropouts in the data arrays.

\subsection{Spectral line selection}

To facilitate data interpretation, lines with known strong blends have been avoided whenever possible. Although the first set of observations (s6159) contains 15 different spectral lines and the second (s6936) 12, there are only 6 lines in common (He I, O III, O IV, O v, Mg IX and $\mathrm{Mgx}$ ). The other lines in s6936 are too weak to be usable, but s6159 offers 8 more lines with sufficient signal-to-noise ratio to be reliably analyzed.

A list of all the 14 spectral lines retained for our analysis is given in Table 1. Their formation temperatures have been taken from Landini \& Monsignori Fossi (1990). Lines from optically dense regions require special consideration when determining the formation temperature, although this is unlikely to be the case for coronal holes. 


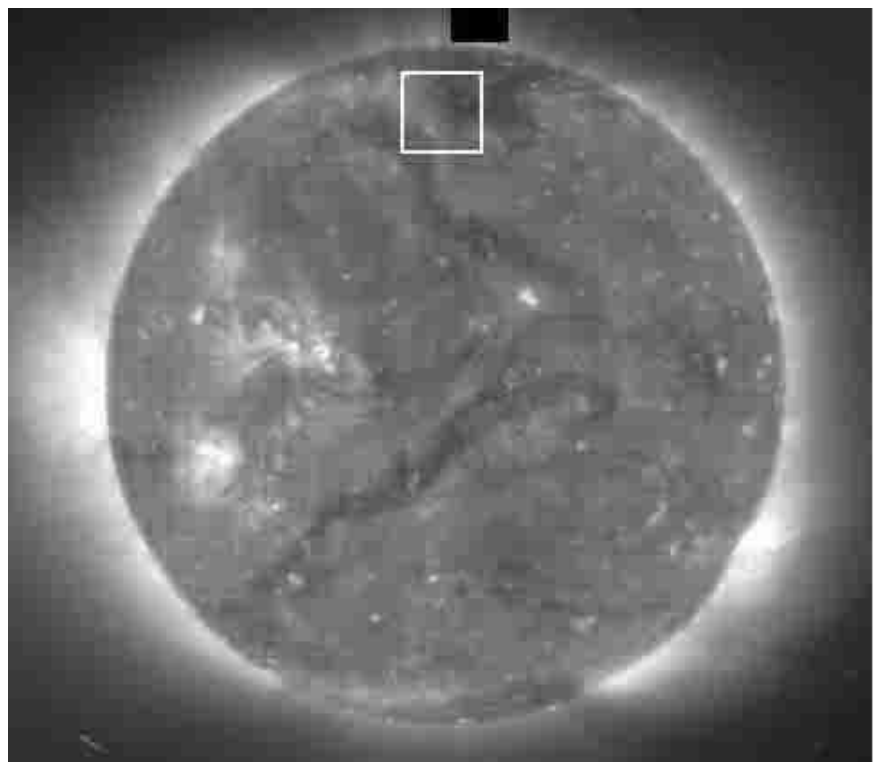

Fig. 1. Image of the Sun as seen by EIT, Fexir $195 \AA$, on December 15th, 1996. The CDS raster for dataset s6159r00 is superimposed.

Table 1. Analyzed spectral lines with formation temperature, the number of Gaussian components used for the fit and the dataset providing these lines.

\begin{tabular}{|c|c|c|c|c|}
\hline Ion & $\begin{array}{l}\text { Wavelength } \\
{[\AA]}\end{array}$ & $\begin{array}{l}\text { Temperature } \\
{[\mathrm{K}]}\end{array}$ & $\begin{array}{c}\text { Fitted } \\
\text { Gaussians }\end{array}$ & Obs. \\
\hline $\mathrm{He}_{\mathrm{I}}$ & 584.33 & $3.90 \times 10^{4}$ & 1 & $\mathrm{a}, \mathrm{b}, \mathrm{c}$ \\
\hline He II & 607.56 & $9.14 \times 10^{4}$ & 2 & $\mathrm{a}$ \\
\hline O III & 599.59 & $1.20 \times 10^{5}$ & 1 & $\mathrm{a}, \mathrm{b}, \mathrm{c}$ \\
\hline O IV & 554.52 & $1.69 \times 10^{5}$ & 3 & $\mathrm{a}, \mathrm{b}, \mathrm{c}$ \\
\hline Ne IV & 543.88 & $1.96 \times 10^{5}$ & 1 & $\mathrm{a}$ \\
\hline $\mathrm{Ov}$ & 629.73 & $2.60 \times 10^{5}$ & 1 & $\mathrm{a}, \mathrm{b}, \mathrm{c}$ \\
\hline $\mathrm{Ne}_{V}$ & 572.33 & $3.00 \times 10^{5}$ & 1 & $\mathrm{a}$ \\
\hline Ne VI & 562.80 & $4.19 \times 10^{5}$ & 2 & $\mathrm{a}$ \\
\hline Ne viI & 558.60 & $5.82 \times 10^{5}$ & 2 & $\mathrm{a}$ \\
\hline $\mathrm{Cax}$ & 557.76 & $7.50 \times 10^{5}$ & 2 & $\mathrm{a}$ \\
\hline Mg VIII & 315.01 & $8.00 \times 10^{5}$ & 1 & $\mathrm{a}$ \\
\hline Mg IX & 368.06 & $9.95 \times 10^{5}$ & 2 & $\mathrm{a}, \mathrm{b}, \mathrm{c}$ \\
\hline $\operatorname{Mg} x$ & 624.94 & $1.35 \times 10^{6}$ & 1 & $\mathrm{a}, \mathrm{b}, \mathrm{c}$ \\
\hline Si XII & 520.67 & $2.25 \times 10^{6}$ & 1 & $\mathrm{a}$ \\
\hline
\end{tabular}

a: s6159r00; b: s6936r00; c: s6936r01.

\subsection{Data analysis}

Prior to the analysis, we "flagged" the pixels, assigning them either to a coronal hole, the quiet Sun, or a "buffer" zone forming the interface between the two. Pixels assigned to the buffer zone are not analyzed further. The quiet Sun was itself subdivided into two zones. The quiet Sun small $\mu$ zone contains the pixels lying within the range of heliospheric angles covered by the coronal hole pixels, while the quiet sun large $\mu$ zone contains all quiet Sun pixels located closer to Sun center.

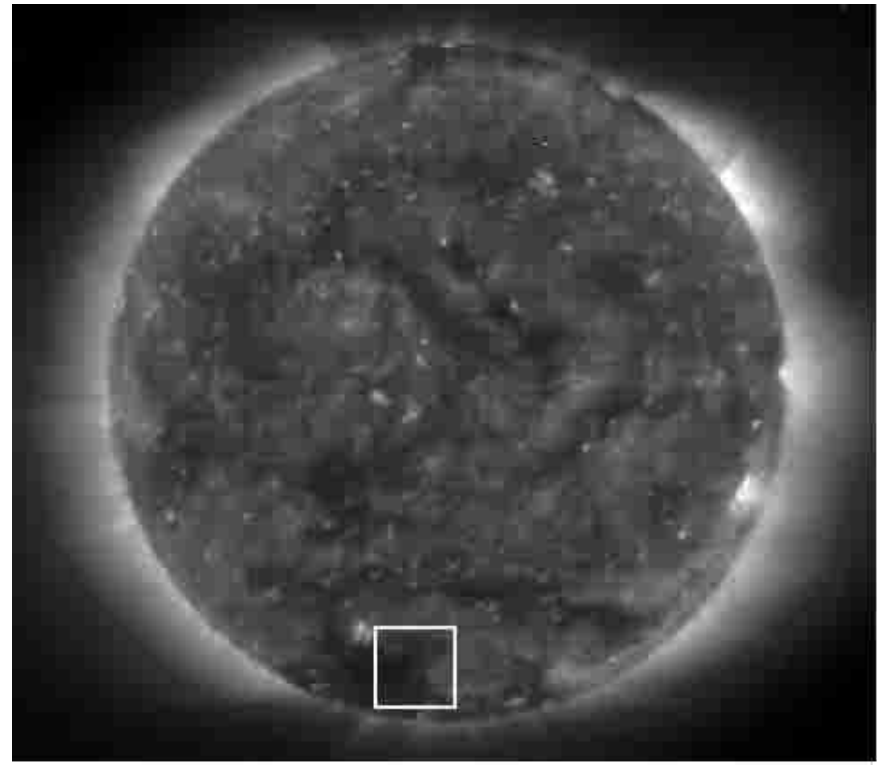

Fig. 2. Same as Fig. 1, on February 9th, 1997. The CDS raster for dataset s6936r00 is superimposed. Dataset s6936r01 was recorded at the same position.

Line parameters are determined from fits of a Gaussian plus linear background to the spectrum at each spatial position. In cases in which a dominant line has one or more minor blends a multiple Gaussian fit plus background has been employed to separate the contributions of the blends. The number of Gaussian components used for the fit to each spectral line is listed in the fourth column of Table 1. Initial fits to profiles from individual spatial pixels turned out to be too unreliable due to noisy profiles. Hence we binned a number of spatial pixels before Gaussian fitting. The number of pixels in the bins depends on the data set, the spectral line and the zone. It varies between $2 \times 2$ binned pixels for the strong lines and $6 \times 6$ pixels in the coronal hole for some coronal lines which were very weak there.

The integrated intensity, shift and width parameters of the selected lines were obtained at each binned spatial position from the best-fit Gaussians. The reference value for the shift has been chosen for each line separately as the mean value of the wavelength of the peak of the Gaussian profiles averaged over the whole area. Thus the choice of reference for the shifts is not related to an absolute wavelength scale and has no influence on the results. The influence of solar rotation has been taken into account by subtracting its value from the obtained line shifts.

Not many authors have measured wavelength shifts using CDS. The accuracy of line shift values obtained with CDS is lower than with SUMER for example, due to the lower spectral resolution of the instrument.

Nevertheless, a careful analysis does allow relatively accurate determination, as a number of investigations have demonstrated. For example, Brynildsen et al. (1998) studied active regions using 10 spectral lines covering a wide range of ionization temperatures. They estimate the 

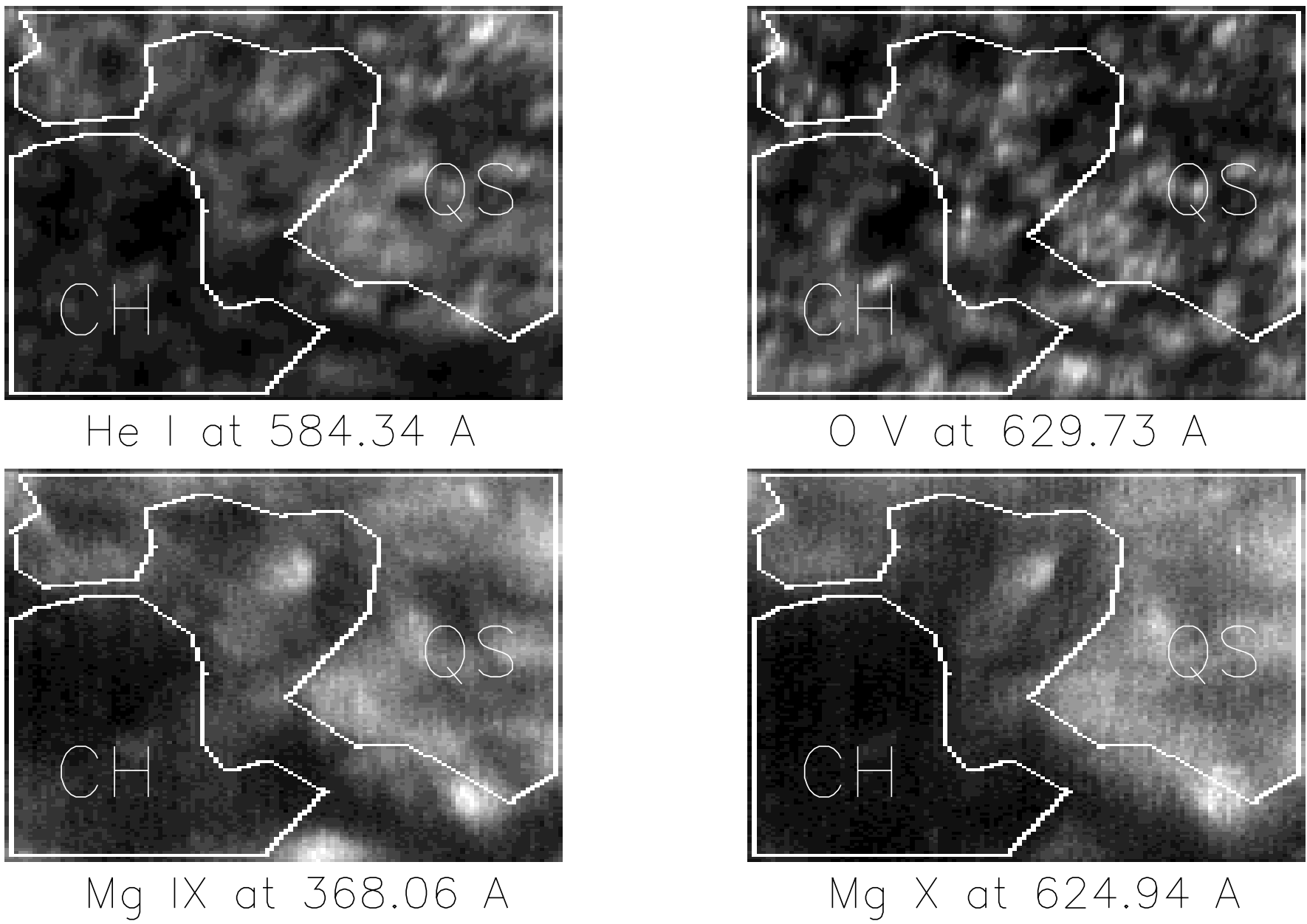

Fig. 3. Intensity images derived from CDS spectra of four of the observed emission lines ( $\mathrm{He}, \mathrm{Ov}, \mathrm{Mg} \mathrm{IX}$ and $\mathrm{Mg} \mathrm{x})$. The regions of coronal hole $(\mathrm{CH})$ and quiet Sun $(\mathrm{QS})$ selected for analysis are outlined.

accuracy in the line-of-sight velocity determinations to be $5 \mathrm{kms}^{-1}$ for HeI and $\mathrm{Ov}, 10 \mathrm{kms}^{-1}$ for $\mathrm{MgIX}$, and $15 \mathrm{kms}^{-1}$ for O III, O IV, Nevi and Fexvi. Brekke et al. (1997) analyzed line shifts in active region loops, in particular for $\mathrm{O} v 629 \AA$, and estimated the uncertainty in the measured shifts to be $\approx 10 \mathrm{~km} \mathrm{~s}^{-1}$. Hence we also do not expect a higher accuracy from the analysis of individual line profiles, in particular due to the low intensity in coronal holes. However, by averaging together the profiles at numerous spatial locations prior to fitting and by binning together the deduced parameters, this accuracy is improved. Inspite of this, we expect that the accuracy will be lower than from similar analyses.

As explained in the appendix, a trend in the shifts and the widths of the spectral profiles was noticed over the image on the detector. The applied correction to the wavelength shift and the line width parameters is described there.

Figure 3 shows intensity images in spectral lines of He I, $\mathrm{O} v, \mathrm{Mg} \mathrm{IX}$ and $\mathrm{Mg}$ x, for the data set s6936. The zones identified as coronal hole $(\mathrm{CH})$ and quiet Sun (QS) are outlined. In Fig. 4 average profiles of these spectral lines are plotted, with the solid curve indicating the average in the coronal hole and the dotted line that in the quiet Sun small $\mu$ region.

In Fig. 5 we plot the number of data points (spatial pixels) in the three analyzed zones as a function of limb distance. All data sets are counted (s6159r00, s6936r00 and s6936r01). The vertical scale expresses the number of pixels in a $3^{\prime \prime}$ band. The overlap of the quiet Sun small $\mu$ region curve with the quiet Sun large $\mu$ region curve is due to the fact that the coronal hole boundary is located at a different heliocentric angles in different data sets. It can be seen from this figure that the distribution of points in the coronal hole zone and the quiet-Sun small- $\mu$ zone is similar. The average limb distances of all the pixels assigned to the three zones are indicated by the vertical lines. Hence we expect little bias in our analysis due to differences in $\mu$ between quiet Sun and coronal hole data.

Examples of the variation of the line parameters (intensity, wavelength shift and line width) along the Sun's radius are presented for $\mathrm{Mg} \mathrm{x}(624.94 \AA)$ and O v $(629.73 \AA)$ in Fig. 6 . The different number of data points for the two lines is due to the different amount of spatial binning applied to them. The center-to-limb brightening in $\mathrm{O} v$ is noticeable, although it is distorded by the presence 

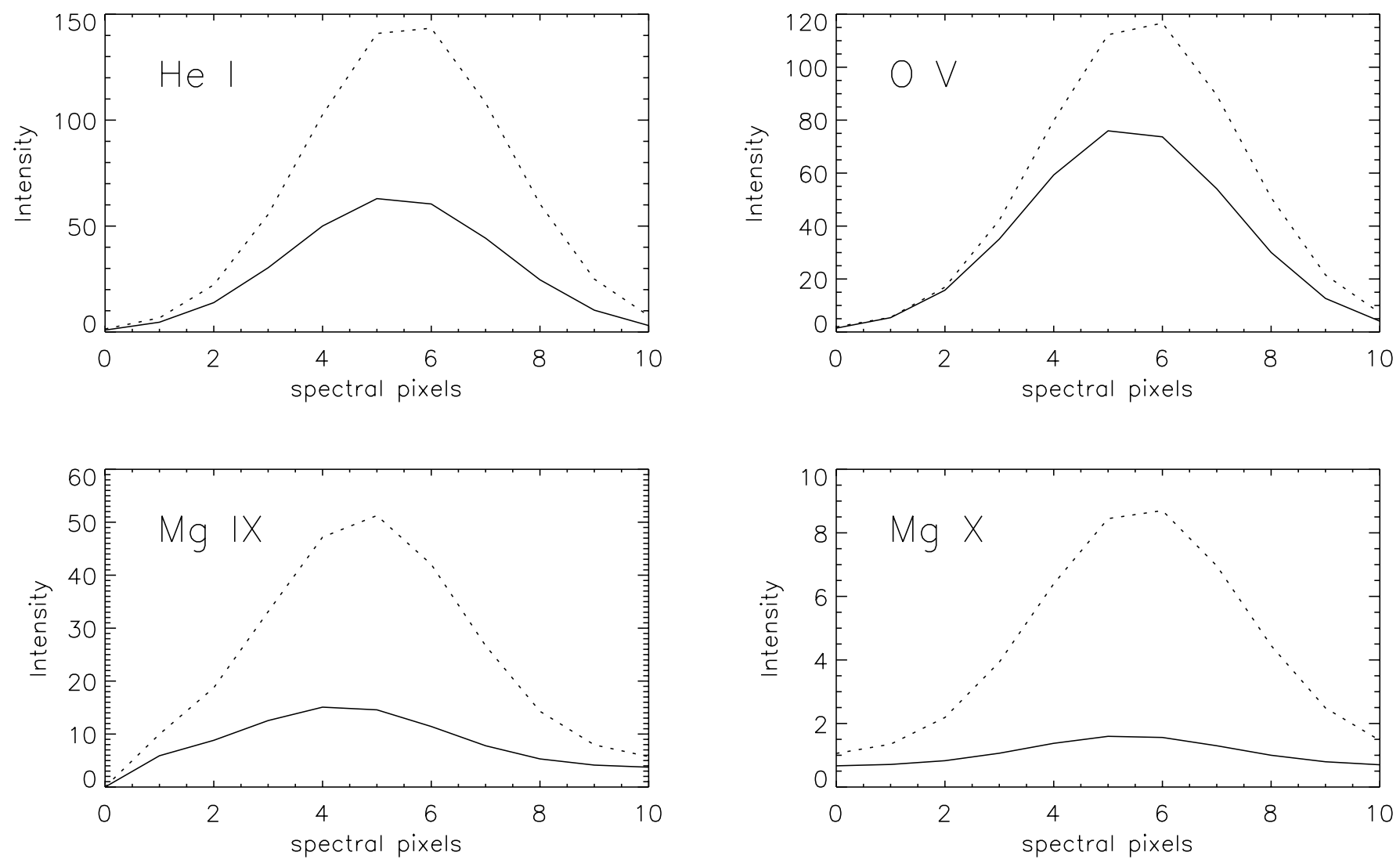

Fig. 4. Best-fit spectral profiles averaged over the coronal hole region (solid line) and over the quiet Sun region (dotted line) at the same average heliospheric angle.

of spatial inhomogeneities (network). Also, the lines are on average blue-shifted inside the coronal hole (relative to the average outside the hole) and - in the case of $\mathrm{O} v$ at least - somewhat broader. The scatter of the points of a given type of feature provides an indication of the statistical uncertainty in the deduced line parameters. These values may appear more precise on the basis of Fig. 6 than values quoted in the literature, but it must be recalled that the plotted values are heavily binned. Systematic errors may of course also be present. Examples are excessive line widths due to instrumental broadening, and residual systematic line shift errors still present after removal of the spectral shift over the detector and along a scan. We estimate that when averaged over the coronal hole the statistical error in the line shift varies between 0.3 and $3 \mathrm{~km} \mathrm{~s}^{-1}$, depending on the strength of the line and to a smaller extent on the number of Gaussians needed to fit it. Judging the systematic error is more difficult, but we expect them to lie below $5 \mathrm{~km} \mathrm{~s}^{-1}$.

We need to remark on the blended line pair of Cax $557.76 \AA$ and Ne vir 558.60 $\AA$. A double Gaussian fit has been applied to the blend and the profile parameters of both lines are analyzed. These did not show larger scatter than those of other lines. In Fig. 7 we illustrate how differently sensitive the two lines react to the coronal hole. The intensity of Cax is significantly reduced inside the hole, while Ne VII shows no apparent difference. Note that these two lines have relatively similar formation temperatures.

\section{Results}

\subsection{Comparison of line parameters}

Figure 8 displays the relative difference between the averaged intensity in the coronal hole region and in the quiet Sun small $\mu$ region as a function of line formation temperature. It was calculated using the expression $2 \cdot\left(I_{\text {coronal hole }}-I_{\text {quiet Sun }}\right) /\left(I_{\text {coronal hole }}+I_{\text {quiet }}\right.$ Sun $)$. Lines formed above $6-7 \times 10^{5} \mathrm{~K}$ show a markedly (up to an order of magnitude) lower intensity in a coronal hole than in the quiet Sun. The transition from lines not influenced (in intensity) by the coronal hole and those significantly weakened by it is surprisingly narrow in temperature. Whereas the Ne VII line formed at $5.8 \times 10^{5} \mathrm{~K}$ remains unaffected by the coronal hole, Ca X (at $\left.7.5 \times 10^{5} \mathrm{~K}\right)$ weakens to less than half its quiet-Sun strength (cf. Fig. 7). It is also roughly in this temperature range that the brightness structure of the Sun changes, with cooler lines showing the network, while hotter ones give indications of loops (Matthews \& Harra-Murnion 1997).

The two helium lines form an exception. Due to their high excitation energy they do not reflect thermal equilibrium behaviour (Andretta \& Jones 1997) and exhibit 


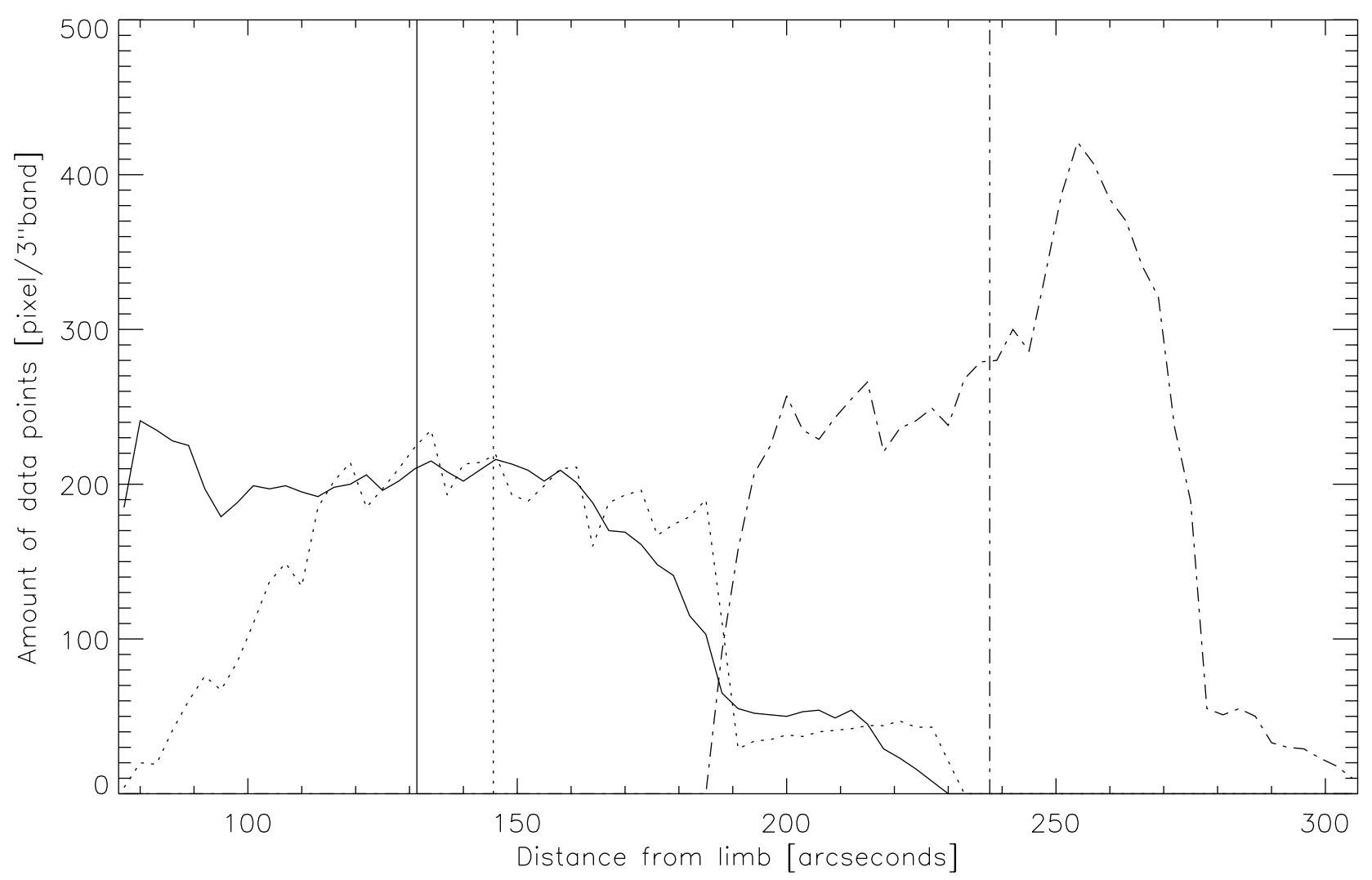

Fig. 5. Distribution of all available data points relative to the radial distance to the solar limb, expressed in arcseconds. The solid line shows the data points located in the coronal hole region. The dotted line shows the points belonging to the quiet Sun region at small $\mu$ (corresponding roughly to the $\mu$ of the coronal hole region) while the dash-dotted line represents the quiet Sun at larger $\mu$.

coronal characteristics to some extent (Feldman et al. 2000). There is no indication of the enhanced intensity of the cooler lines within the coronal hole. This supports the view that such an enhanced intensity, present in the data previously analyzed by Stucki et al. (2000a), is mainly an artefact of the limited statistics from which those data suffered.

The difference between the line widths observed inside the coronal hole and those observed in the small $\mu$ quiet Sun region ( $W$ coronal hole $-W$ quiet Sun $)$ is plotted versus formation temperature in Fig. 9.

According to Lemaire et al. (1999) and Stucki et al. (2000a), the spectral lines are on average broader inside the coronal hole. This increase in line width, which indicates higher non-thermal velocities inside coronal holes, is not so clearly visible in the current data. This may be due to the lower spectral resolution of CDS compared with the SUMER spectrometer, with which the data analyzed by the other authors were recorded. Note also that the differences in line width are small compared to the line width itself, which achieves values of around $150 \mathrm{~km} \mathrm{~s}^{-1}$ in CDS data.

If we consider Fig. 9 more carefully, however, we note that for a formation temperature below $6 \times 10^{5} \mathrm{~K}$ the lines are indeed on the whole somewhat broader in the coronal hole, while above this temperature the opposite is the case. Hence, also for the line width there may be a transition at around the same temperature at which coronal holes become visible in intensity. Observations with higher spectral resolution covering the whole temperature range are required to settle this question. Since SUMER mainly samples the lower temperature range our result is consistent with the findings of Lemaire et al. (1999), who found Ne VIII $770.43 \AA$ to have the same width in quiet Sun and coronal holes, and Stucki et al. (2000a), whose data indicate that Fe XII $1242 \AA$ is narrower in holes.

Figure 10 displays the difference between the wavelengths observed in the coronal hole region and the ones observed in the small $\mu$ quiet Sun region. It shows a blueshift relative to the quiet Sun at all temperatures. The trend with temperature previously found by Stucki et al. (2000a) cannot be recognized. The limited spectral resolution of CDS and the remaining systematic biases in the CDS data (Sect. 2.4 and Appendix A) are probably the main factors responsible for this difference. Nevertheless, the lines formed at high temperatures, sampled only by CDS, show shifts lying in the same range as the cooler lines, similar to those recorded by SUMER. This implies that there is no dramatic increase (or decrease) of the outflow velocity in coronal holes relative to transition region values at coronal temperatures (compared to the quiet Sun). 
$\mathrm{Mg} \times$ at $624.94 \mathrm{~A}$
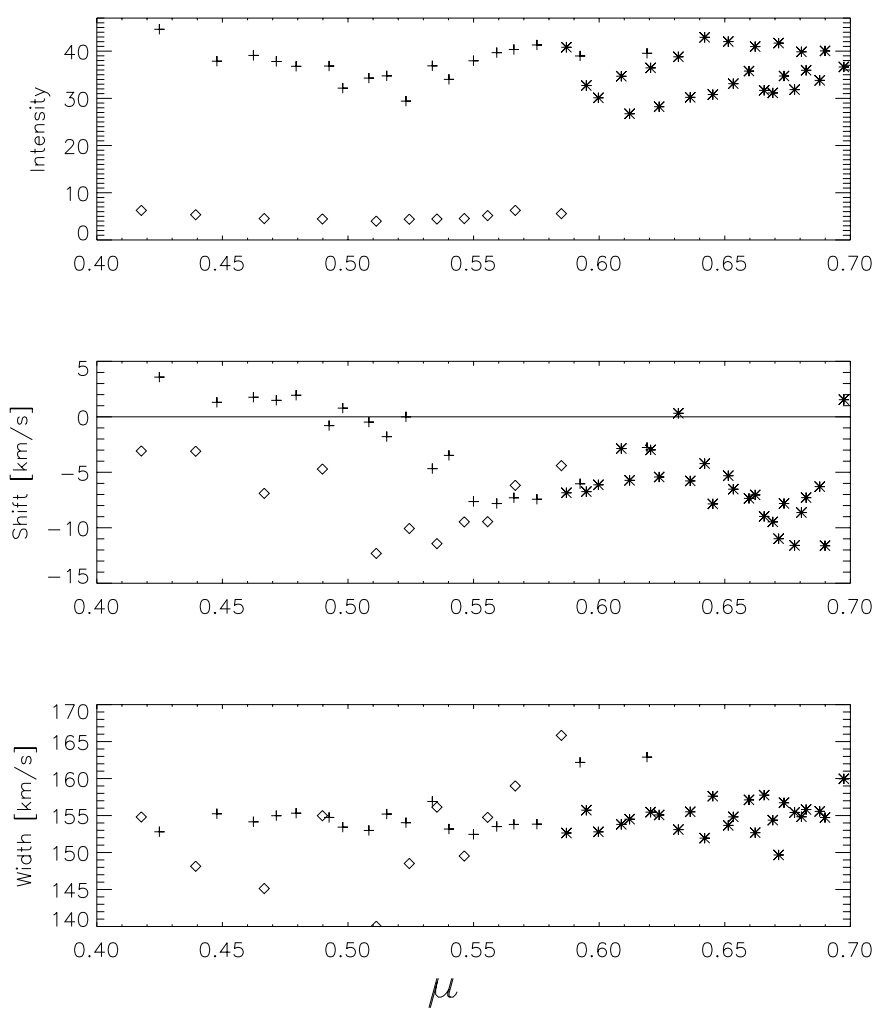

$\mathrm{O} V$ at $629.73 \mathrm{~A}$
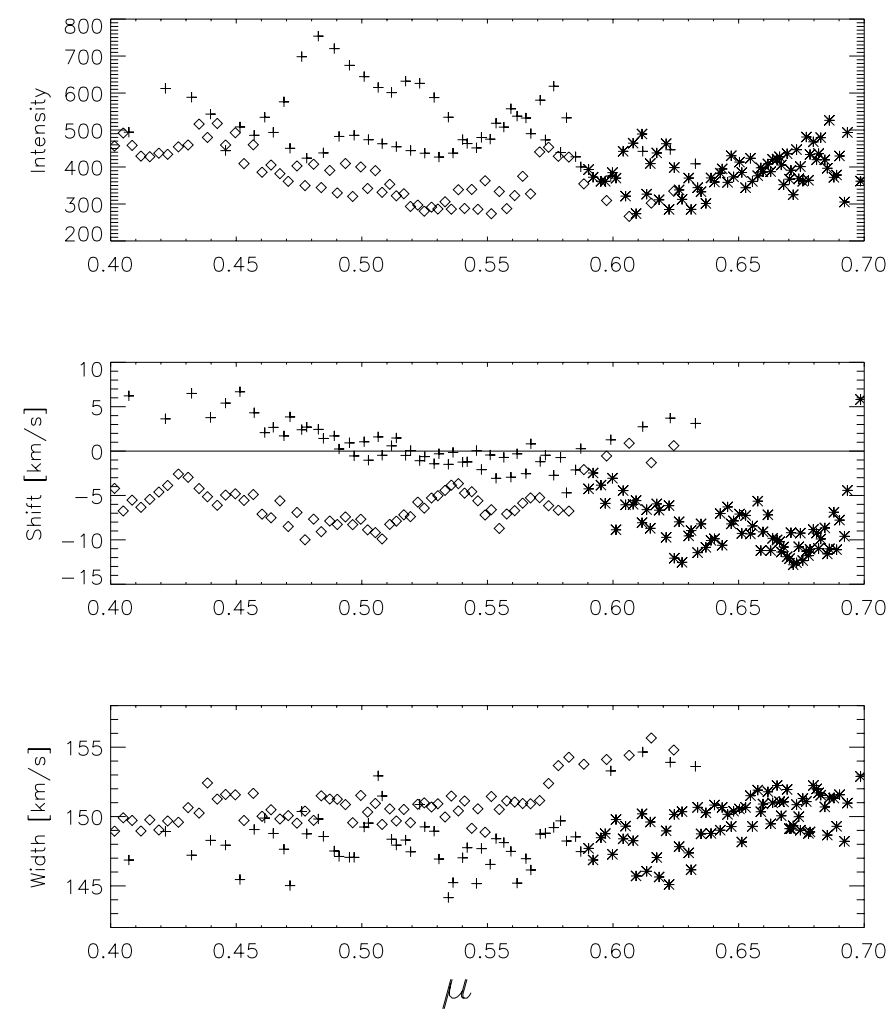

Fig. 6. Examples of line parameters (integrated intensity, shift and width) of the spectral profiles of Mg x at $624.94 \AA$ (left) and O v at $629.73 \AA$ (right) vs. $\mu=\cos \vartheta$, where $\vartheta$ is the heliocentric angle. The diamonds represent the coronal hole region, the crosses the small $\mu$ quiet Sun region and the stars the large $\mu$ quiet Sun region. The integrated intensity is expressed in units of Photon-events/spat.pixel/s. The data presented in these figures were binned in two ways. First, neighbouring spatial pixels were binned as described in Sect. 2.4. Then, to avoid overcharged plots, we binned datapoints with similar $\mu$ values together. For $\mathrm{Mg} \mathrm{x}$, we used bins of 15 datapoints, while bins of 30 datapoints were used for $\mathrm{O} \mathrm{v}$.

\subsection{Distribution of intensities}

Investigations of the quiet Sun in the EUV with Skylab (Huber et al. 1974) indicated that the intensities of EUV features in quiet Sun areas follow a distribution in which there is a strong peak at relatively low intensity and a tail extending to several times the peak intensity. This tail is even more pronounced for areas outside coronal holes. For chromospheric and transition region EUV spectral lines, the higher intensities have been identified with the bright network structures, while the lower intensities of the distribution are related to the supergranule cell interiors (Reeves 1976; Reeves et al. 1976). More recently, the distribution of intensities within transition region images using SOHO has been investigated by several authors (e.g. Lemaire et al. 1997; Dammasch et al. 1999; Pauluhn et al. 2000; Stucki et al. 2000a, with the SUMER spectrograph; Gallagher et al. 1998, with the CDS spectrograph). The new observations confirm older results, but also reveal new insights. Thus the core of the distribution is more sharply peaked in transition region lines and the distribution is best described by a log-normal function.

In our study, we concentrated on comparing the distribution of intensities in a coronal hole and in the quiet Sun. To this end, we plotted the intensity histograms for four representative lines belonging to $\mathrm{He}$ I, O III, O v and $\mathrm{Mg} \mathrm{x}$, in Figs. 11 to 14. In each figure we displayed the histogram for the coronal hole data (small $\mu$ ), for the corresponding quiet Sun data at small $\mu$ and finally for the quiet Sun at large $\mu$. The transition-region lines O III $599.59 \AA$ and O V $629.73 \AA$ exhibit no significant difference in the intensity distribution (Figs. 12 and 13). The center-to-limb variation in the quiet Sun is responsible for the difference between the dashed and the dotted curves, especially for $\mathrm{O} \mathrm{v}$. Both the typical coronal line $\mathrm{Mg}$ x $624.94 \AA$ and the chromospheric He I $584.34 \AA$ line, whose intensity is sensitive to coronal radiation, show not just a lower average intensity within the coronal hole, but also a narrower intensity distribution (Figs. 11 and 14).

We calculated the ratio of mean intensity to width of intensity distribution for all spectral lines. Interestingly, this ratio is smaller in coronal holes than in the quiet Sun. The difference of the ratio in coronal holes to the ratio in quiet Sun is more important for coronal spectral lines. This indicates that although the intensity decreases inside coronal holes, the spatial fluctuations of the intensity are not decreasing proportionately. This agrees with previous studies (Lemaire et al. 1997; Dammasch et al. 1999; Pauluhn et al. 2000; Stucki et al. 2000a; Gallagher et al. 1998). 


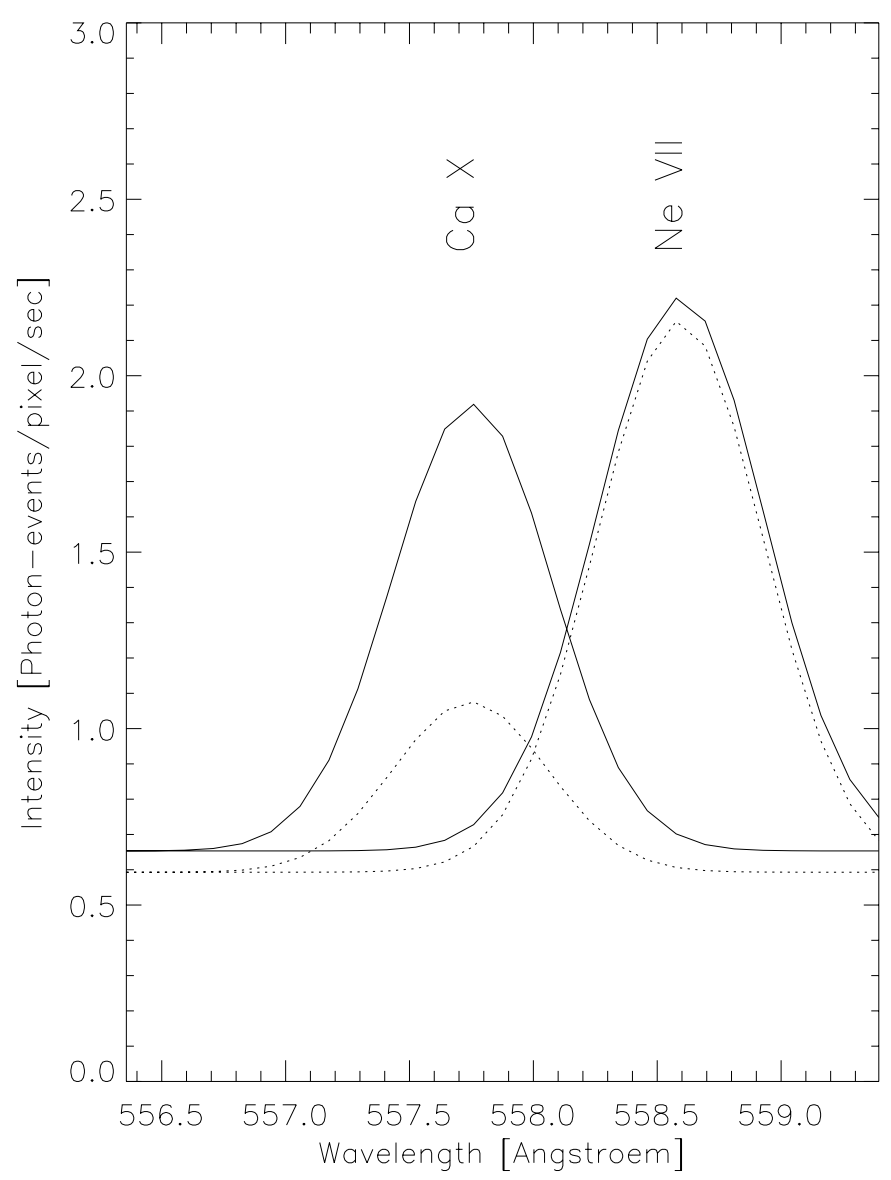

Fig. 7. Averaged Gaussian fits to the spectral profile of the doublet Cax / Nevir. The solid lines refer to the average quiet Sun profiles while the dotted curves represent the coronal hole profiles.

\subsection{Correlations between shifts and intensities}

Here we study the possible relation between line position (Doppler shift) and intensity of the lines showing the chromospheric network. A similar study has been carried out previously with the SUMER instrument (Stucki et al. $2000 \mathrm{~b}$ ). For transition region lines between $2 \times 10^{4}$ and $2 \times 10^{5} \mathrm{~K}$, it showed a trend of increasing blueshift with increasing intensity of the network inside coronal holes compared to the quiet Sun. An open question is whether the trend is maintained for even hotter lines. We are aware that CDS is not an ideal instrument for such a study and we do not expect the results to be of equal quality. Nonetheless, the behaviour of the hotter lines is important for an understanding of the source regions of the fast solar wind. Since all lines are recorded simultaneously with CDS, we compared the shifts of all lines formed above $3 \times 10^{5} \mathrm{~K}$ with the intensity of the O v $629.73 \AA$ line.

In Fig. 15, the correlation coefficients between line shifts and intensities of all the spectral lines in our sample are plotted versus their formation temperature. The few chromospheric lines available in our data set show the typical behaviour of larger redshift in the network (i.e. positive correlation coefficient) for both coronal-hole and
quiet-Sun regions found by Stucki et al. (2000b). Most transition-region lines show the same trend in the quiet Sun, but the opposite trend inside coronal holes, confirming that indeed the blue shift in the coronal hole areas is related with the chromospheric network. The new finding of our investigation is that the coronal lines, at temperatures above $10^{6} \mathrm{~K}$, display positive correlation coefficients in both the quiet Sun and in coronal holes. This result is unexpected and we need to check its reliability.

Due to their low intensities in coronal holes and the consequent extensive binning, the correlations for the coronal lines are less reliable than the rest, being based on less points. To obtain an idea of the reliability of the results summarized in Fig. 15, we have also considered the slopes of regression lines placed through the line shift vs. intensity data points. The signs of these slopes agree in most cases with the signs of the regression coefficients. The slopes of the linear regressions are on average reliable at the $3 \sigma$ (coronal hole) and $4.1 \sigma$ (quiet Sun) level. The median values of these numbers are $1.8 \sigma$ (coronal hole) and $2.3 \sigma$ (quiet Sun), also for the coronal lines. Hence, the result appears correct, but we nevertheless encourage further investigations with improved statistics to confirm or refute it.

When correlating line shifts versus intensity, with both quantities taken from the same spectral line, we find a positive correlation in the coronal hole for the 5 hottest lines $\left(T_{\mathrm{e}} \gtrsim 7.5 \times 10^{5} \mathrm{~K}\right)$. Since we expect the brightness of these lines in a coronal hole to outline polar plumes rather than the network, this result confirms the work of Wilhelm et al. $(1998,2000)$ which shows higher outflow speeds in the interplume regions than in the plumes themselves (cf. Patsourakos \& Vial 2000).

\section{Conclusions}

We derived parameters of a set of EUV spectral lines observed with CDS on SOHO in coronal hole and quiet Sun regions on the solar disk. The aim is to determine in what sense coronal holes differ from the normal quiet Sun, as revealed by EUV emission lines. The present study is an extension of previous investigations by Stucki et al. (2000a,b) to higher temperatures and larger spatial samples, at the cost of lower spectral resolution. Comparison of intensities in quiet Sun and coronal hole areas confirmed results previously obtained by Huber et al. (1974), up to the maximum plasma temperature of $2 \times 10^{6} \mathrm{~K}$ that our data samples. Averaged over the coronal hole there is still some emission, even at this high temperature, although it is an order of magnitude weaker than in the normal quiet Sun. These averaged intensities are reliable at the 15 to $20 \sigma$ level.

If we assume for simplicity that the emission geometry is the same in the coronal hole and the normal quiet Sun then the emission measurements imply that the density of gas at over two million degrees is on average only a factor of 3 lower in coronal holes than in the normal quiet Sun. If, however, the emission is restricted to individual 


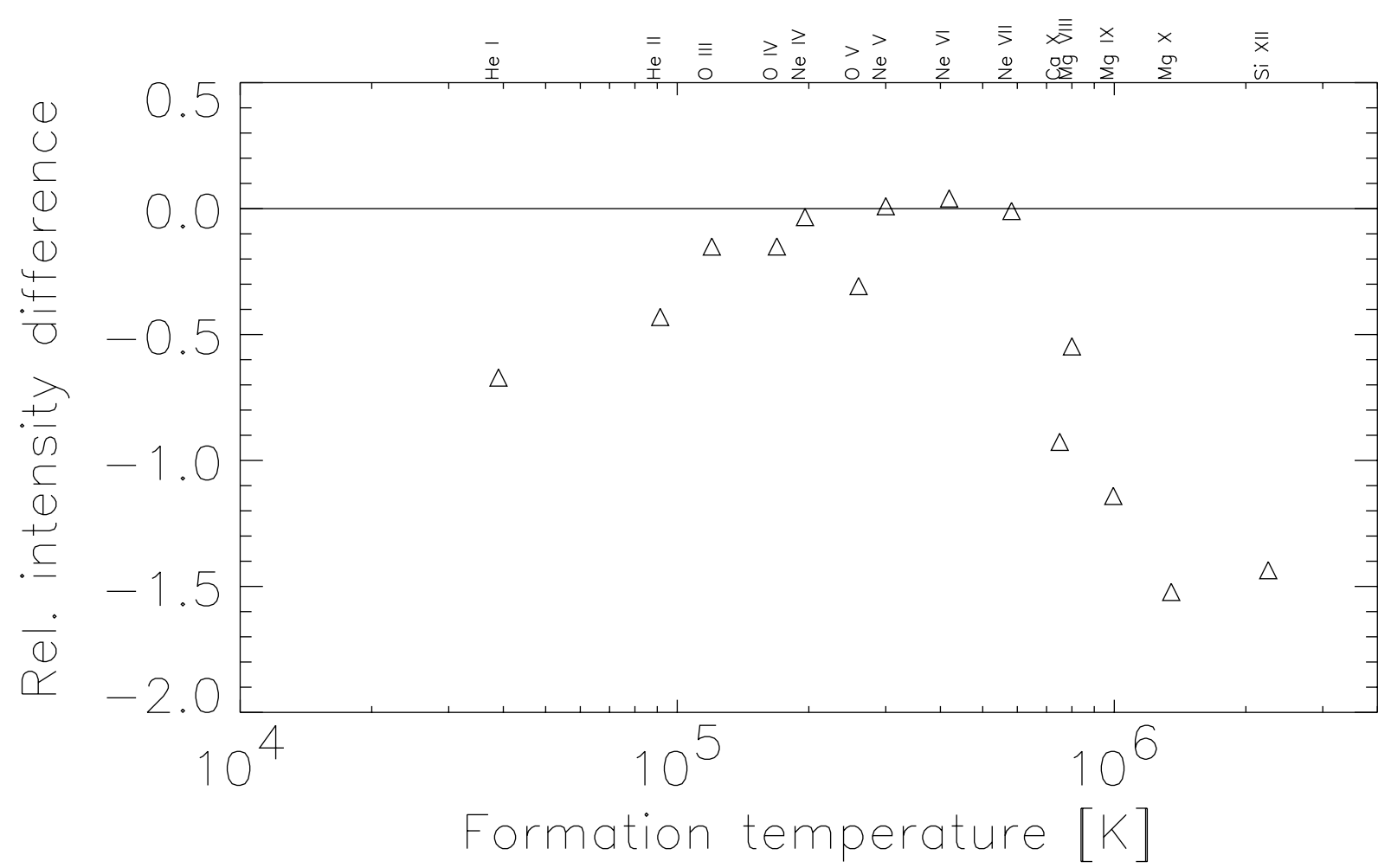

Fig. 8. Relative integrated intensity difference between the coronal hole and the quiet Sun small- $\mu$ zone vs. line formation temperature.

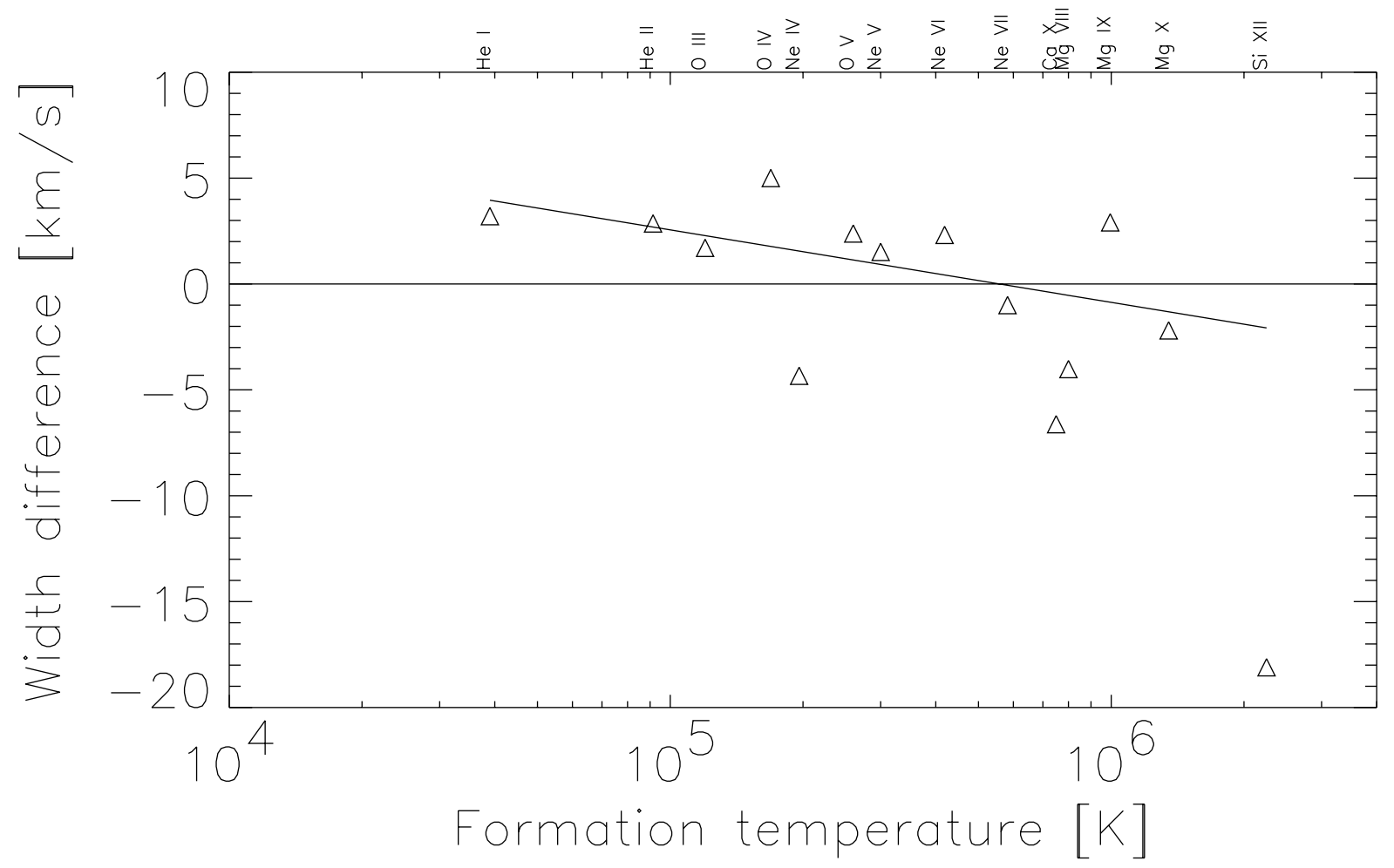

Fig. 9. The difference between the line width observed in the coronal hole and in quiet Sun regions vs. line formation temperature. The solid line shows a weighted linear regression fit to the datapoints.

loops, in which the density is the same in both regions, then the volume filling factor of such loops must decrease by a factor of 10 from quiet Sun to coronal hole.
We also find that the coronal hole starts manifesting itself in line intensity rather abruptly at temperatures above $6-7 \times 10^{5} \mathrm{~K}$. 


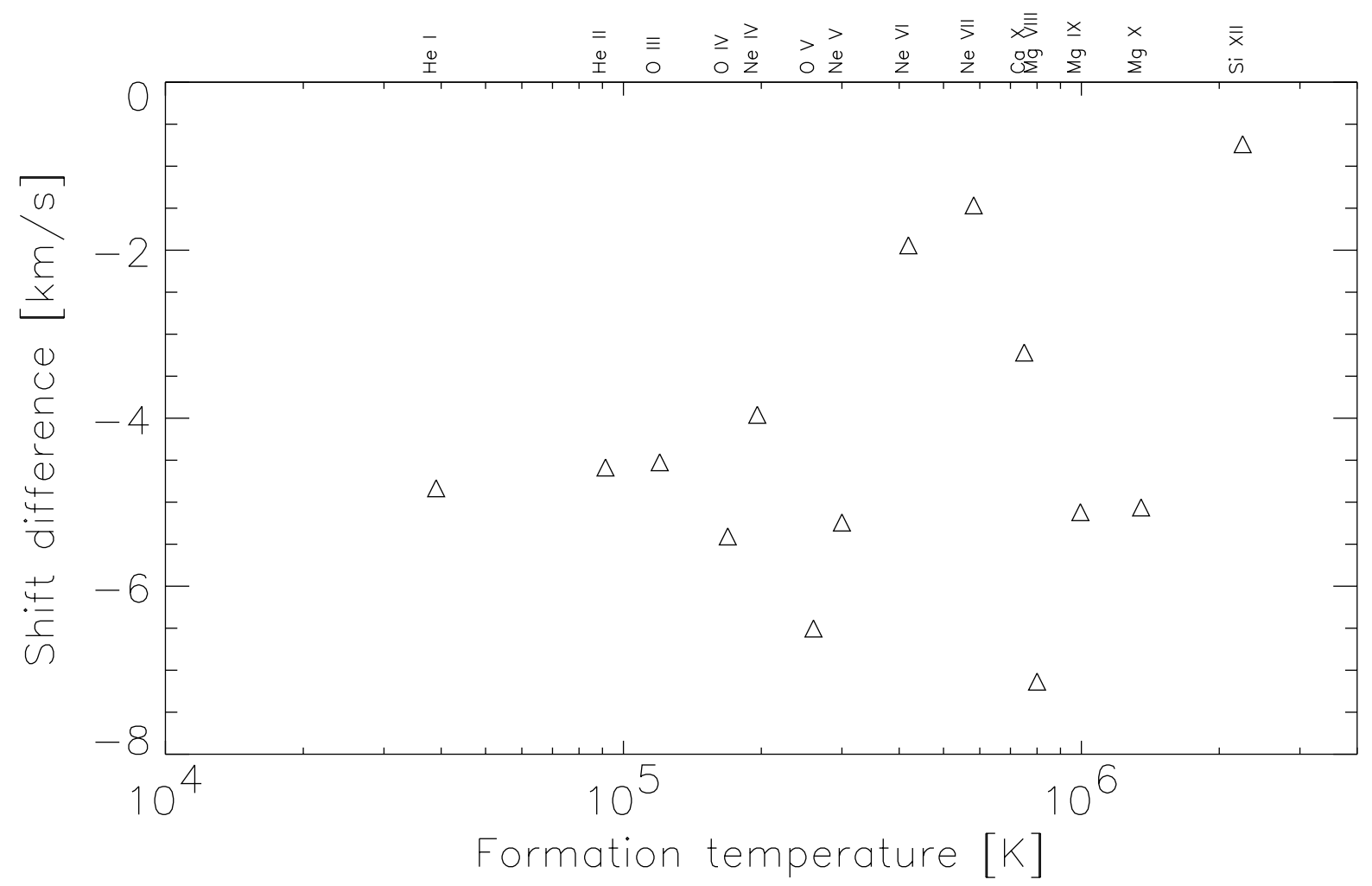

Fig. 10. Wavelength difference in velocity units vs. formation temperature. The "wavelength difference" represents the difference between the wavelength observed in the coronal hole and the one observed in quiet Sun at the same $\mu$. Negative shifts signify blueshifts.

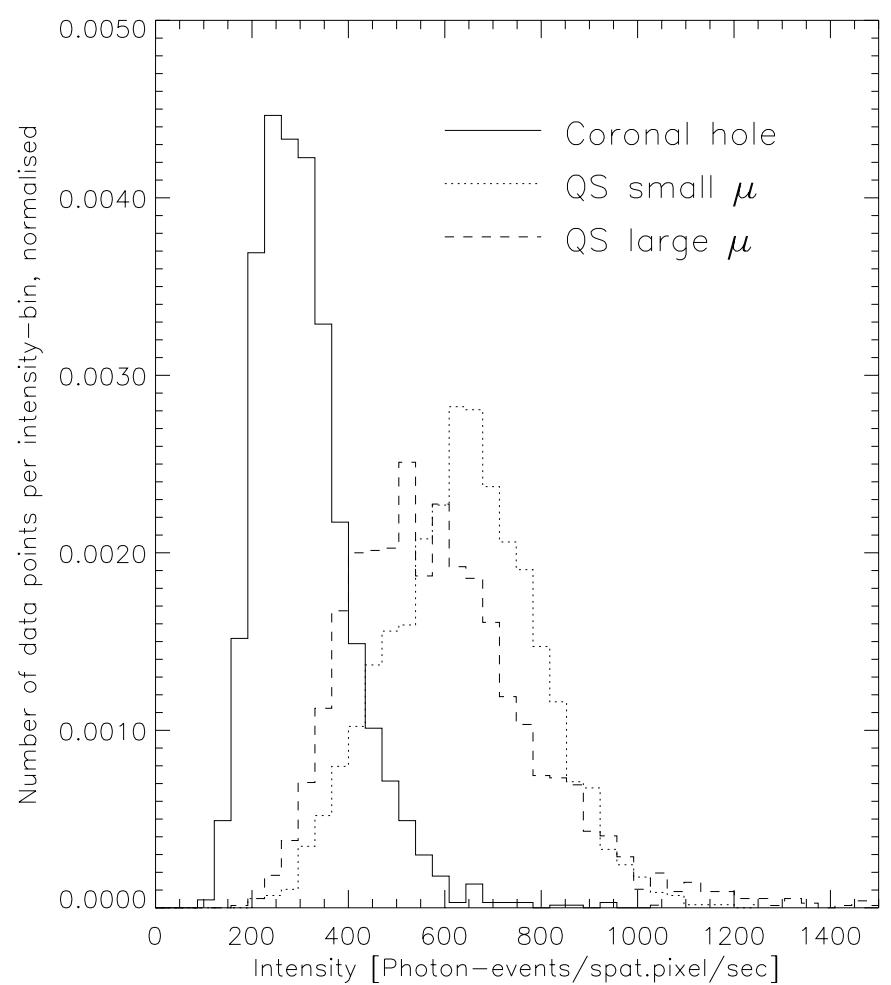

Fig. 11. Integrated intensity histogram of the He I line at 584.33 A. Solid line: coronal hole data (at small $\mu$ ). Dotted line: quiet Sun data at small $\mu$. Dashed line: quiet Sun data at large $\mu$.

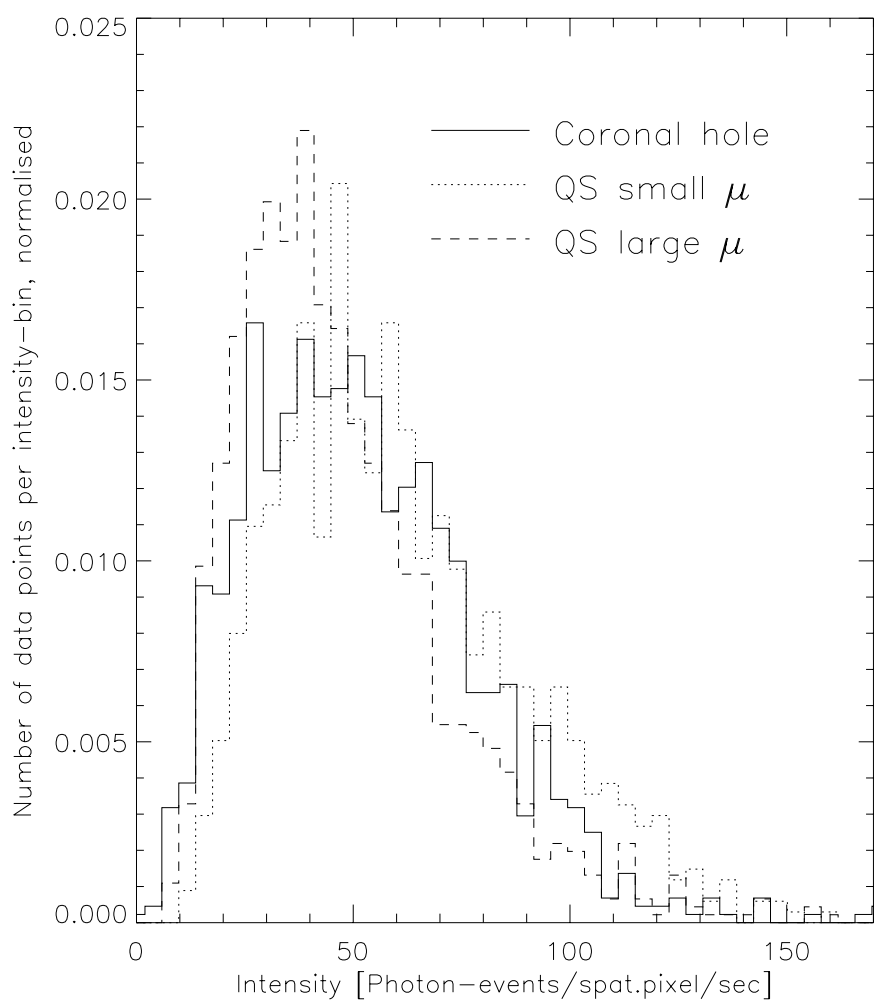

Fig. 12. Same as Fig. 11 for O III at $599.59 \AA$. 


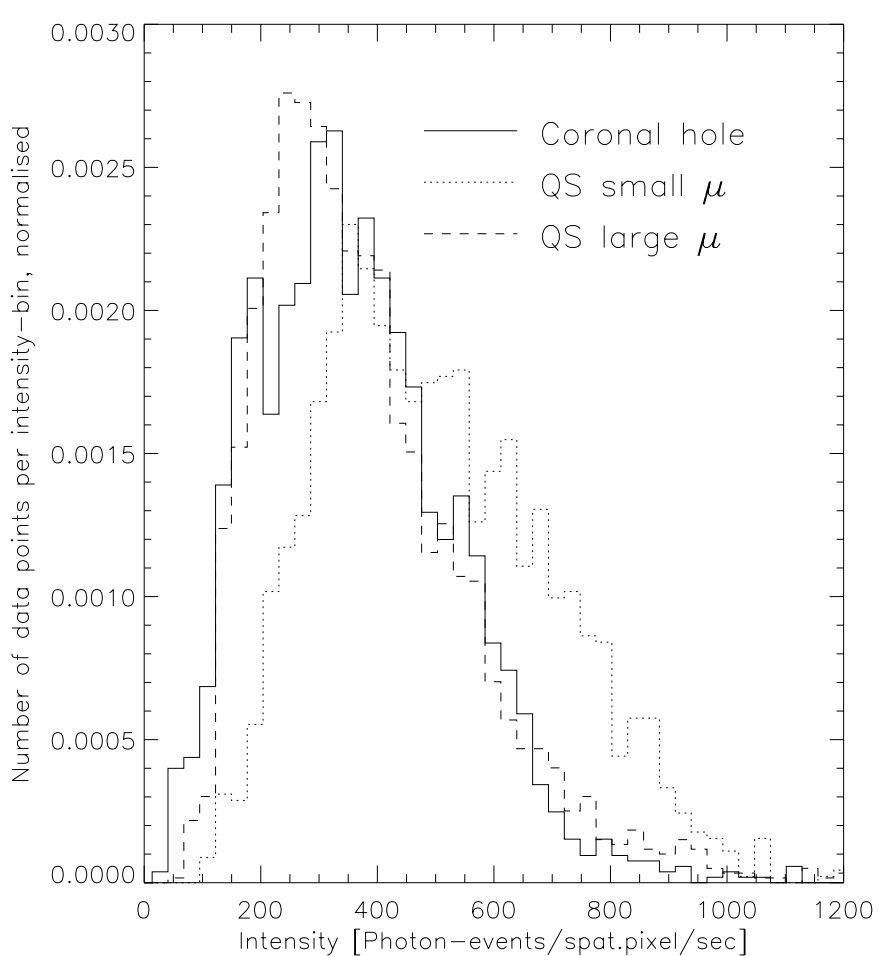

Fig. 13. Same as Fig. 11 for $\mathrm{O}_{\mathrm{v}}$ at $629.73 \AA$.

Results on line widths and shifts are less reliable than those involving the line intensity due to the low spectral resolution of CDS. Nevertheless, the CDS line width measurements confirm the earlier SUMER results for chromospheric and transition-region lines, while the truly coronal lines exhibit a lower line width in coronal holes (such lines were not sampled earlier).

The greater spatial coverage due to the raster scan mode leads to a better statistical basis for this result. Furthermore we only cautiously identify a general blueshift of the lines inside coronal hole areas relative to quiet Sun areas. Within the accuracy of the present data these are compatible with earlier SUMER results. The hotter lines not observed earlier exhibit line shifts that are similar to those of cooler lines.

The correlation of the line shift inside coronal hole and quiet Sun with the intensity of the chromospheric network could also be confirmed for transition region lines, whereas at plasma temperatures higher than $10^{6} \mathrm{~K}$ the correlation of line shift with the chromospheric network seems to be inverted. Data sampling high temperatures, but obtained at high spectral resolution are required to improve upon the present results.

Acknowledgements. SOHO is a mission of international cooperation between ESA and NASA. CDS was built and is operated by a consortium led by the Rutherford Appleton Laboratory and including Mullard Space Science Laboratory, the NASA Goddard Space Flight Center, Oslo University and the Max-Planck-Institute for Extraterrestrial Physics, Garching. We would like to express particular thanks to R. Harrison and the CDS team, to M. C. E. Huber and J. O. Stenflo. This work was partly supported by the Swiss

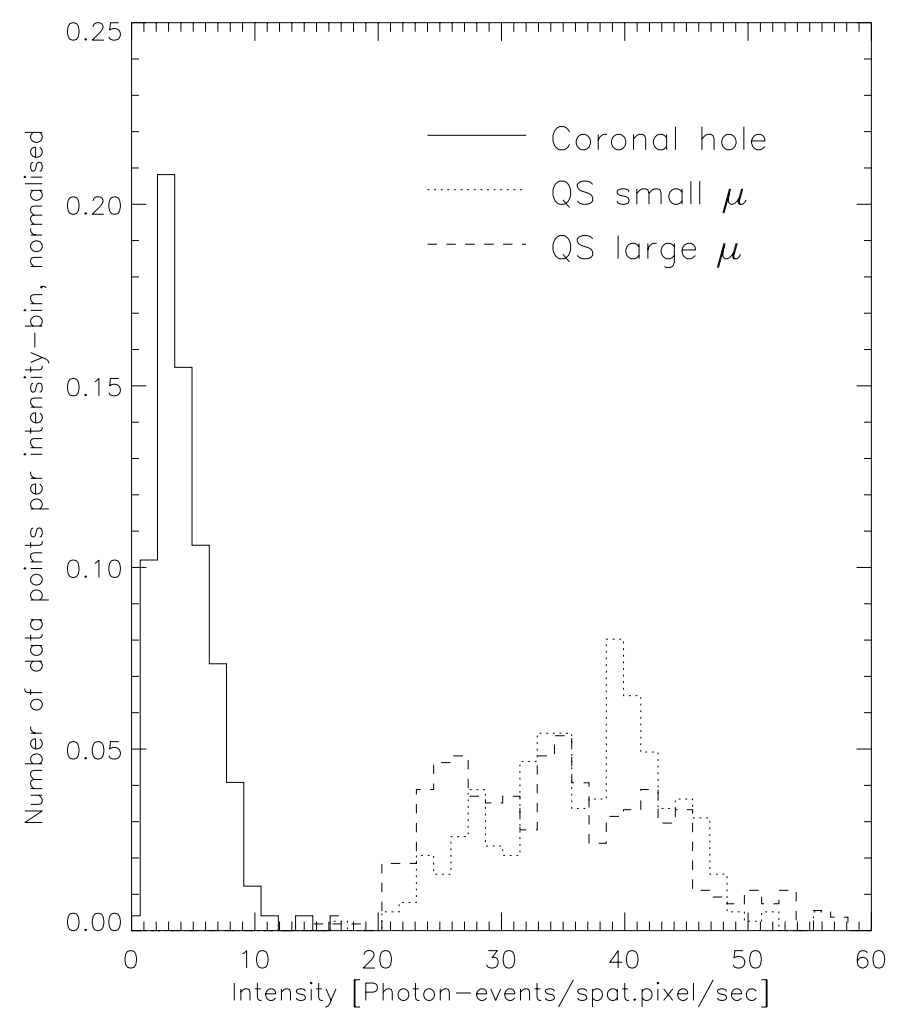

Fig. 14. Same as Fig. 11 for $\mathrm{Mg} \mathrm{x}$ at $624.94 \AA$.

National Science Fundation, grant No. 20-55456.98, and by a grant from the ETH-Zürich which are gratefully acknowledged.

\section{Appendix A: Spectral line shift and width corrections on CDS data}

When inspecting images of individual exposures we noticed that the wavelength shifts exhibit an anomalous behaviour. Such an effect had been earlier seen by e.g. Pike (1996, 1998) and Haugan (1998). Since the standard CDS software for correcting this problem turned out to be insufficiently accurate for our purposes and our study is based to a large extent on the analysis of line shifts and widths, we have searched for a more detailed and accurate correction.

The problem arises from the geometrical distortion of the spectra (rotation and tilt). The parameters that the standard software developed to correct for these effects uses for the corrections (line tilt and scan mirror shift) are only average representations of the effect and are not applicable to individual datasets if the analysis requires high accuracy.

Early on in the mission it was also found that the wavelength zero point of the spectra changed with temperature and, even though the optical bench was stabilised, small temperature changes caused by different previous pointings still have an effect. They probably even have "second order" effects in that the size of the line tilt and mirror shift are temperature dependent and so almost always vary from raster to raster. This can be easily shown, since after applying the standard corrections there are usually 


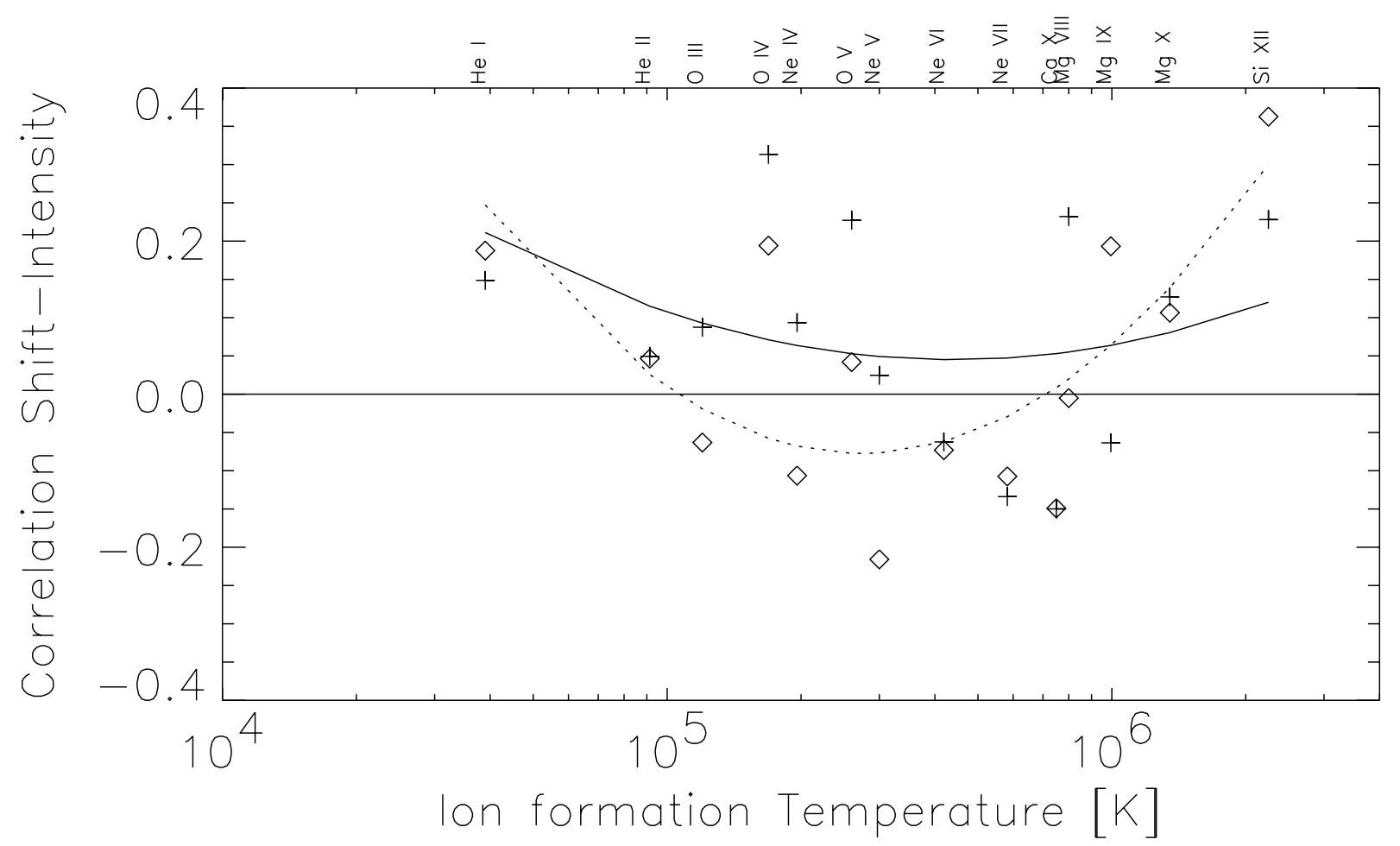

Fig. 15. Correlation coefficient between wavelength shifts and intensities, versus the ion formation temperatures. Diamonds: coronal hole. Crosses: quiet Sun. The curves are quadratic fits to coronal hole (dashed) and quiet Sun (solid) data.

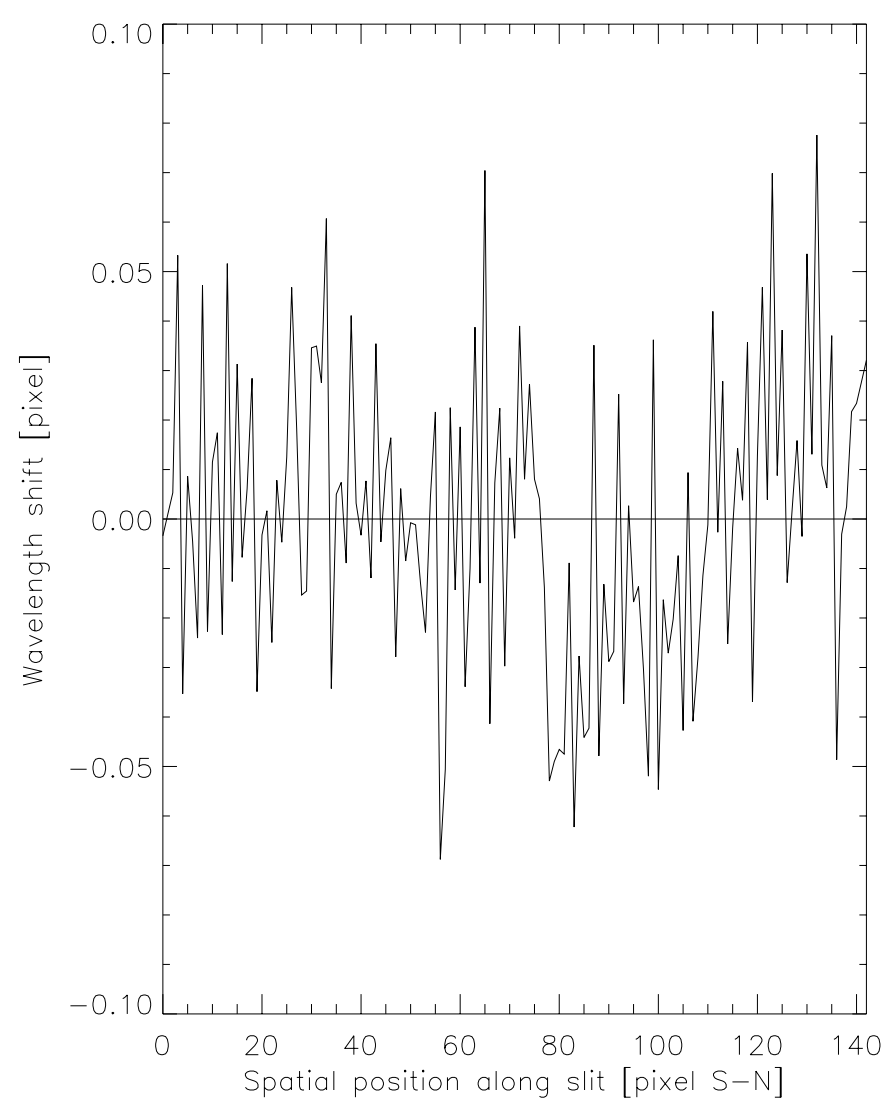

Fig. A.1. High-frequency fixed pattern correction. This pattern was determined using $\mathrm{O}_{\mathrm{v}} 629.73 \AA$ data. It is a onedimensional array along the slit, from south to north. still systematic trends both along the slit and in the scan direction in velocity maps.

When correcting for these effects, we found that the best results are obtained by proceeding in two steps. First, one subtracts a high-frequency fixed pattern map (see Fig. A.1). Afterwards, 2-dimensional surfaces are fitted to each individual velocity map to eliminate the linear $\mathrm{N} / \mathrm{S}$ (slit axis) and quadratic E/W (scan axis) trends. In our case, since we are analysing images containing regions with very different characteristics (coronal hole and nonhole quiet Sun) on different parts of the image we expect that correction surfaces created for each image would be biased. For this reason, we have created correction surfaces using an image of the quiet Sun taken at disk center and containing as little solar structure as possible, and applied the same correction surface to all our data, after testing it on samples obtained at the poles.

\section{A.1. High-frequency fixed pattern correction}

A correction map for the high-frequency pattern of the velocities, using Ov $629.73 \AA$ data has been constructed. First, the velocity maps from several months of disk-center synoptic scans were averaged. Then a 2-dimensional surface was subtracted from it, which left a high-frequency fixed pattern trace. This trace can now be subtracted from any data set. This fixed pattern appears to be time independant and applicable to other spectral lines. Figure A.1 shows the deduced fixed pattern (along the slit, $\mathrm{S}$ to $\mathrm{N}$ ). 

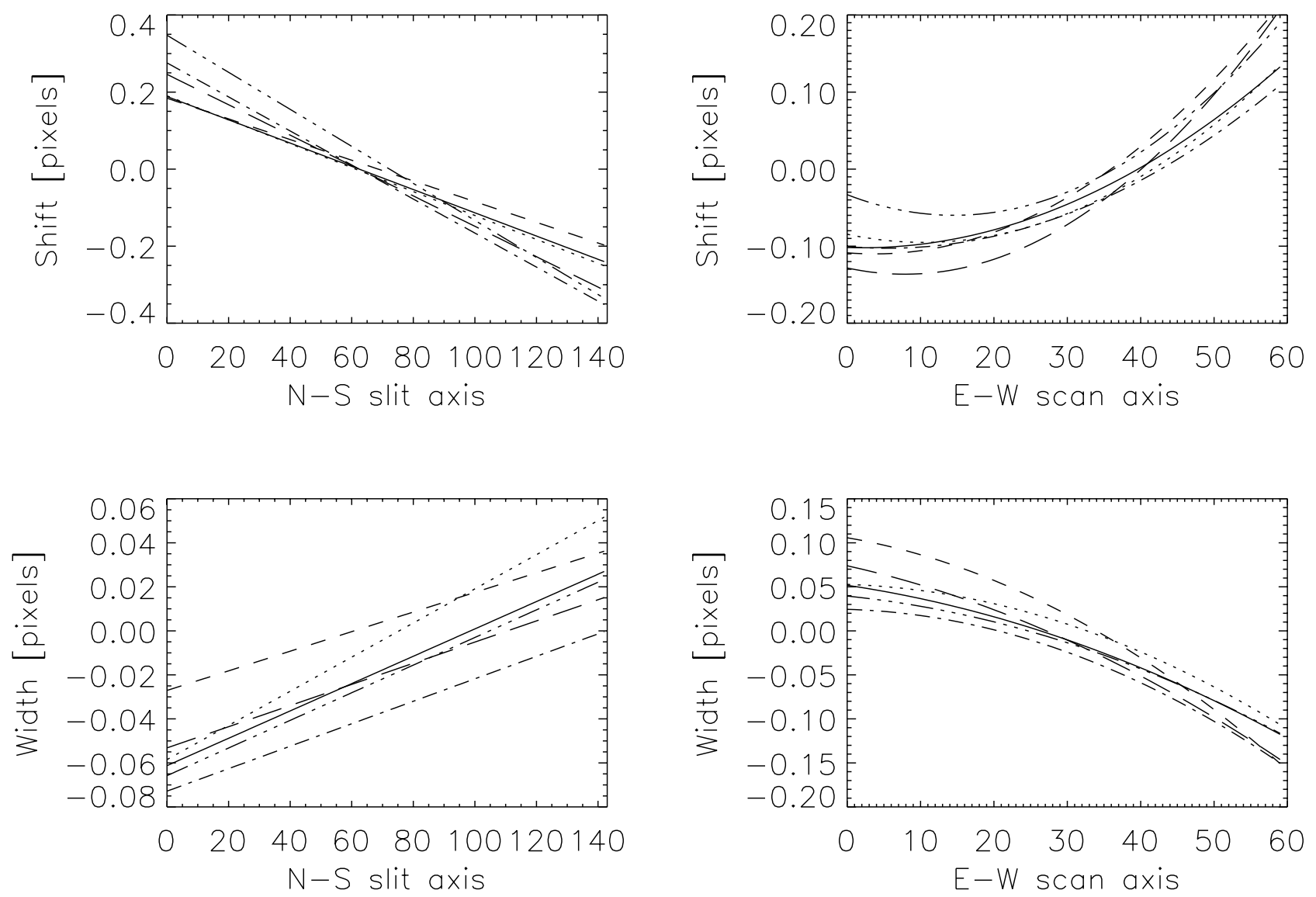

Fig. A.2. Line shift and width trends along the slit (left panels) and the scan axis (right panels) for NIS-2. The upper two plots show the behaviour of the spectral line shift while the lower two plots show the behaviour of the line width, for He I $584.33 \AA$ and Ov $629.73 \AA$ A. Solid line: He I averaged for the observation s7104r00 to s7104r04. Dotted line: He i from s6159r00. Dashed line: He r from s6936r00. Dash-dotted line: Ov from s7104r00. Dash-triple-dotted line: Ov from s6159r00. Long dashes: Ov from s6936r00.

\section{A.2. Observations used in the analysis of the phenomenon}

For the analysis of the trends in line shifts and widths we used three sets of data. The first two sets are the data used for the study presented in this paper (s6159r00 and s6936r00); see Sect. 2.1. To avoid bias introduced by solar structures or limb proximity effects, we have chosen a third dataset, s7104. This set covers $240^{\prime \prime}$ by $240^{\prime \prime}$ at disk center and exhibits only quiet sun with homogeneous solar supergranulation. Furthermore, several rasters were made successively at the same position, which allows us to test the time/temperature dependance of the distortion. The dataset s7104 was recorded on February 26, 1997, using slit 5 (secondary slit, $4^{\prime \prime}$ by $240^{\prime \prime}$ ) with an exposure time of $15 \mathrm{~s}$. It includes 3 strong and unblended EUV emission lines: He I $584.33 \AA$ (NIS-2), Mg Ix $368.06 \AA$ (NIS-1) and O v $629.73 \AA$ (NIS-2), which are all used for the trend analysis. All datasets were cleaned for cosmic rays, corrected for CCD readout bias, flatfielded, calibrated and interpolated over missing pixels. Of course, no spectral line tilt or rotation correction was applied.

Once the data were reduced, a Gaussian fit was applied to the spectrum at every spatial pixel. The line parameters (intensity, shift and width) were retained. The reference value for the shift of each line has been chosen as the mean value of the wavelength of the peak of the Gaussian profiles averaged over the whole area.

\section{A.3. Observed trends}

The line shifts and widths exhibit sizeable trends, stronger even than the effects of the relatively pronounced solar structure differences present in the different windows, including coronal holes. Thus, if we fit a two-dimensional surface to the shift and width trends in both coronal hole observations analyzed in our study, they show an almost identical behaviour, although the coronal holes are present on opposite sides of the detector in the two cases. The effect of the geometrical distortion is of such importance relative to the effect of solar structures that it needs to be meticulously corrected if one wants to obtain reliable results on line shifts and widths using CDS.

Figure A.2 shows cuts through the surfaces fitted to the line shifts and widths for He I $584.33 \AA$ and Ov $629.73 \AA$, on NIS-2 in all three analyzed data sets. The upper plots show the trends in line shift, while the lower plots show the trends in line width. We plot the 

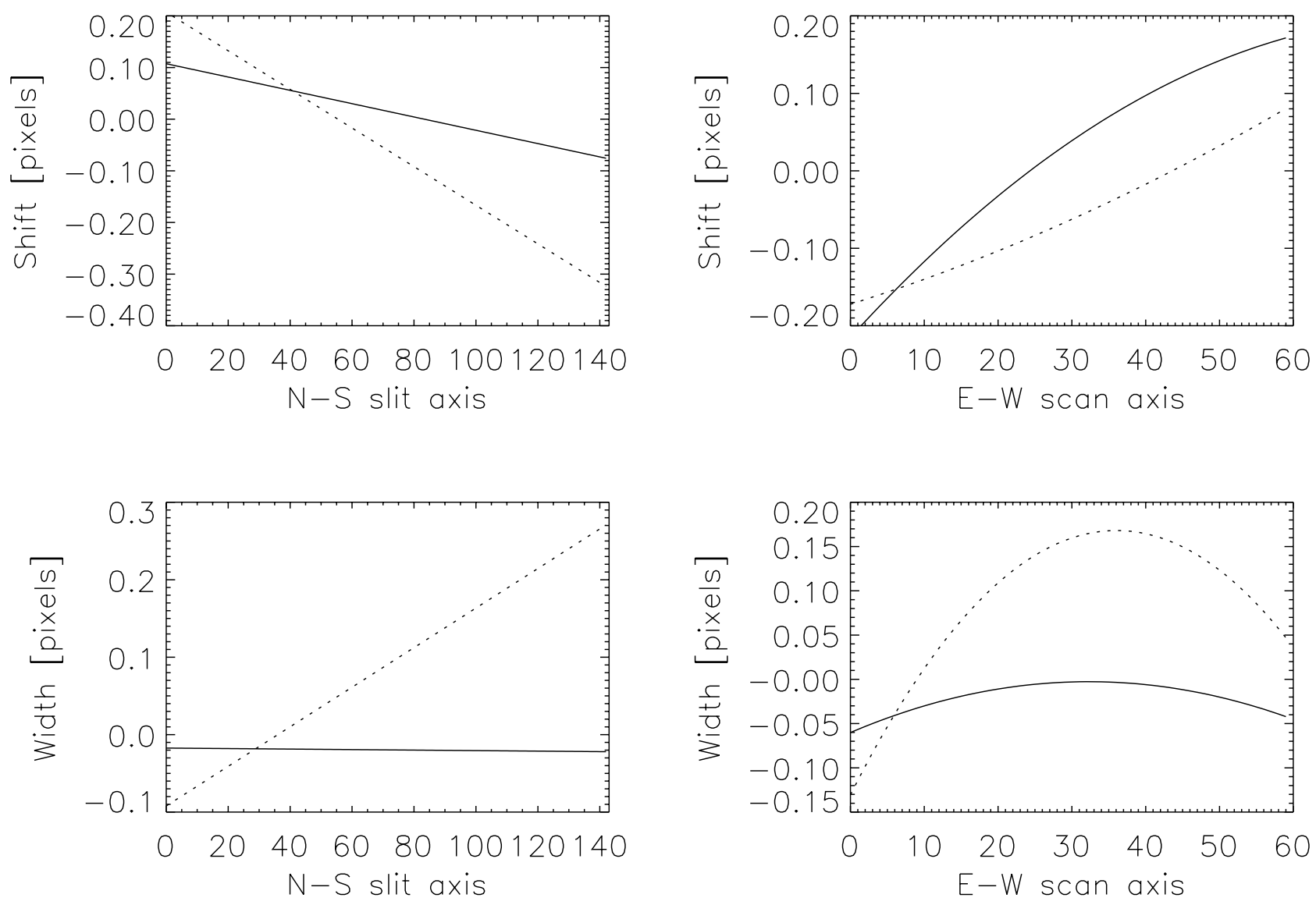

Fig. A.3. Line shift and width trends along the slit and the scan axis for NIS-1. The upper two plots show the behaviour of the spectral line shift while the lower two plots show the behaviour of the line width, for Mg Ix $368.06 \AA$. Solid line: Mg Ix from s7104r00. Dotted line: Mg Ix from s6159r00.

average surface along the N-S slit axis in the frames on the left side and the average along the E-W scan axis in the frames on the right. The trend is best fitted by a surface that is linear along the slit and quadratic in the scan direction. The effect along the scan axis is expected to be temperature- (and therefore time-) dependent, although for the data shown in Fig. A.2 this is not the case to any significant extent (see below).

Figure A.3 shows the same as Fig. A.2, but for NIS-1. This range of the spectrograph includes less strong lines, and we could study the geometrical distortion only with one line, Mg Ix $368.06 \AA$, with two observations: s7104 and s6159. Although less pronounced than on NIS-2, the trend in line shifts can still be recognized. For the widths however, we cannot get conclusive results. This is due to the fact that Mg IX is less strong than the other spectral lines of the dataset and is more coronal-hole sensitive.

To determine if the effect of time and temperature of the instrument need to be taken into account, we studied the trends presented by 5 raster scans observed successively (s7104r00, s7104r01, ... to s7104r04). The difference is negligible. This means that a surface correction determined with the first raster (s7104r00 in this case) may be applicable to the following rasters as well.

\section{A.4. Adopted correction surface for line shifts and widths}

The adopted correction surfaces are given by the following equations ( $X$ being the pixel number on the E-W scan axis, and $Y$ on the N-S slit axis). They are given for a window of 60 by 143 spatial pixels (60 being the number of scan steps and 143 the pixels along the slit direction), but the correction can be easily rescaled for other window dimensions (the slope remains unchanged and the surface is shifted to get an average correction of zero over the whole window).

$$
\begin{aligned}
F_{\text {shift-NIS } 2=} & 0.171-0.00268 \cdot X-0.00384 \cdot Y \\
& +0.0000336 \cdot X \cdot Y+0.0000825 \cdot X^{2} \\
F_{\text {width-NIS2 }}= & -0.0109-0.000775 \cdot X+0.000882 \cdot Y \\
& -0.00000644 \cdot X \cdot Y-0.00000235 \cdot X^{2} \\
F_{\text {shift-NIS1 }}= & -0.226+0.0139 \cdot X+0.000106 \cdot Y \\
& -0.0000482 \cdot X \cdot Y-0.0000677 \cdot X^{2} \\
F_{\text {width-NIS1 }}= & 0
\end{aligned}
$$

This way, the distortion correction is known for every spatial position and can be subtracted from the corresponding line parameters (after the fixed pattern correction has been done, see Sect. A.1). 
Nevertheless, depending on the features present in the observations, one should think about using correction surfaces for each separate dataset. If feasible, the highest accuracy can certainly be achieved by deriving a correction surface from each dataset, or group of closely associated datasets, which is then applied to the dataset itself. If correction surfaces cannot be determined in this way, one should not necessarily use the particular correction surfaces presented here but use instead the CDS standard software. The parameters of that software are based on the analysis of many datasets and may be more appropriate for the general case.

\section{References}

Ahmad, I. A., \& Withbroe, G. L. 1997, Sol. Phys., 53, 397

Andretta, V., \& Jones, H. P. 1997, ApJ, 489, 375

Arnaud, M., \& Rothenflug, R. 1985, A\&AS 60, 425

Bravo, S., \& Stewart, G. A. 1997, ApJ, 489, 992

Brekke, P., Kjeldseth-Moe, O., \& Harrison, R. A. 1997, Sol. Phys., 175, 511

Brynildsen, N., Maltby, P., Brekke, P., et al. 1998, ApJ, 502, L85

Dammasch, I. E., Hassler, D. M., Curdt, W., et al. 1999, Space Sci. Rev., 87, 161

Feldman, U., Schühle, U., Widing, K. G., et al. 1998, ApJ, 505, 999

Feldman, U., Dammasch, I. E., Wilhelm, K. 2000, Space Sci. Rev., 93, 411

Gallagher, P. T., Phillips, K. J. H., Harra-Murnion, L. K., et al. 1998, A\&A, 335, 733

Harrison, R. A., Sawyer, E. C., Carter, M. K., et al. 1995, Sol. Phys., 162, 233

Hassler, D. M., Dammasch, I. E., Lemaire, P., et al. 1999, Science, 283, 810

Haugan, S. V. H. 1998, Sol. Phys., 185, 275

Haugan, S. V. H. 1999, Sol. Phys., 178, 275

Huber, M. C. E., Foukal, P. V., Noyes, R. W., et al. 1974, ApJ, 194, L115
Krieger, A. S., Timothy, A. F., \& Roelof, E. C. 1973, Sol. Phys., 29,505

Landini, M., \& Monsignori Fossi, B. C. 1990, A\&AS 82, 229

Leer, E., \& Holzer, T. E. 1985, in Future Missions in Solar, Heliospheric and Space Plasma Physics, ed. E. Rolfe, \& B. Battrick (ESA Scientific \& Technical Publications Branch, Noordwijk, The Netherlands), 3

Lemaire, P., Wilhelm, K., Curdt, W., et al. 1997, Sol. Phys., 170,105

Lemaire, P., Bocchialini, K., Aletti, V., et al. 1999, Space Sci. Rev., 87, 249

Matthews, S. A., \& Harra-Murnion, L. K. 1997, Sol. Phys., 175,541

McKenzie, J. F., Sukhorukova, G. V., \& Axford, W. I. 1998, A\&A, 330, 1145

Patsourakos, S., \& Vial, J.-C. 2000, A\&A, 359, L1

Pauluhn, A., Solanki, S. K., Rüedi, I., et al. 2000, A\&A, 362, 737

Peter, H., \& Judge, P. G. 1999, ApJ, 522, 1148

Pike, C. D. 1996, unpublished

Pike, C. D., \& Mason, H. E. 1998, Sol. Phys., 182, 333

Reeves, E. M. 1976, Sol. Phys., 46, 53

Reeves, E. M., Vernazza, J. E., \& Withbroe, G. L. 1976, Phil. Trans. R. Soc. London, Ser. A, 281, 319

Stucki, K., Solanki, S. K., \& Schühle, U. 2000a, A\&A, 363, 1145

Stucki, K., Solanki, S. K., \& Schühle, U. 2000b, A\&A, 362, L49

Teriaca, L., Banerjee, D., \& Doyle, J. G. 1999, A\&A, 349, 636

Thompson, W. T. 1998, CDS Software Note, 49

Tu, C.-Y., Marsch, E., Wilhelm, K., \& Curdt, W. 1998, ApJ, 503,475

Warren, H. P., Mariska, J. T., \& Wilhelm, K. 1997, ApJ, 490, L187

Wilhelm, K., Marsch, E., Dwivedi, B. N., et al. 1998, ApJ, 500, 1023

Wilhelm, K., Lemaire, P., Dammasch, I. E., et al. 1998, A\&A, 334,685

Wilhelm, K., Dammasch, I. E., Marsch, E., et al. 2000, A\&A, 353, 749

Wilhelm, K. 2000b, A\&A, 360, 351

Woch, J., Axford, W. I., Mall, U., et al. 1997, Geophys. Res. Lett., 24, 2885 